\title{
Linfoma no Hodgkin primario de mama: reporte de un caso
}

\author{
Gámez Oliva H. ${ }^{1 *}$, Batlle Zamora S. ${ }^{1}$, Blanco González Y. ${ }^{1}$, Córdova I. ${ }^{1}$, Brito García S. ${ }^{1}$, Pérez Blanco V. M. ${ }^{1}$
}

Resumen - El linfoma no Hodgkin primario de la mama es una patología poco frecuente, constituye menos del 0,5\% de todos los tumores mamarios malignos, debido a que carecen de características propias, tanto clínicas, mamográficas como ultrasonográficas. Es difícil el diagnóstico preoperatorio, la citología mediante BAAF tiene mejor rendimiento que el estudio con material congelado ya que este último tiende a confundirse con el carcinoma. En la actualidad se prefiere el tratamiento con quimioterapia tanto para el tratamiento local como para el regional. Presentamos el caso de un linfoma no Hodgkin primario de la mama en una paciente de 72 años.

Palabras clave: linfoma no Hodgkin; mama

Abstract - Primary non-Hodgkin lymphoma of the breast is a rare entity. It represents less than $0.5 \%$ of all breast cancer malignancies. No features at clinical presentation distinguish patients with lymphoma from those with carcinoma of the breast. There are both mammographic and sonographic difficulties to establish the preoperative diagnosis. Contemporary frozen sections can be mistaken with breast carcinoma. In this article we present a case of a primary non-Hodgkin lymphoma of the breast in 72 years old women.

Keywords: Breast; non Hodgkin Lymphoma

Fecha de envío: 16 de octubre del 2015 - Fecha de aceptación: 31 de diciembre del 2015

\section{Introducción}

Los linfomas no Hodgkin (LNH) son un grupo de neoplasias malignas con una localización y comportamiento biológico muy variable. Dentro de su amplio espectro de presentaciones clínicas, existen lesiones de crecimiento muy lento y evolución crónica con buena supervivencia, como los linfomas foliculares de bajo grado, y formas agresivas de crecimiento rápido, que pueden ser leucemizados y tener una evolución letal a corto plazo, como los linfomas linfoblásticos e inmunoblásticos, entre otros. Los LNH primarios de mama representan entre el $0.004 \%$ y el $0.5 \%$ de los cánceres mamarios, y menos del $0.5 \%$ del total de $\mathrm{LNH}$; y constituyen entre el $1.7 \%$ y el 2.2\% de los LNH extraganglionares (Fajoses Gonçalves et al., 2011; Ferbeyre-Binelfa et al., 2013).

El LNH es uno de los diagnósticos posibles ante cualquier lesión sólida de la mama. Se suelen presentar en un rango de edad entre 17 y 90 años, con una media de edad de 55 años, aunque se han descrito casos en niños (Verdecia-Cañizares et al., 2011). Pueden ser unilaterales o bilaterales, y estos últimos evolucionar de forma sincrónica o metacrónica. En la mayoría de los reportes, la mama derecha suele estar más afectada que la izquierda. Hay varios casos reportados en personas del sexo masculino (Ferbeyre-Binelfa et al., 2013).

Las formas clínicas e imaginológicas de presentación pueden ser muy variadas, siendo indispensable realizar una biopsia con estudio de inmunohistoquímica para el diagnóstico definitivo. El diagnóstico diferencial incluye lesiones inflamatorias como la mastitis; idiopáticas, como la enfermedad de Rosai Dorffman; y neoplásicas, como los carcinomas poco diferenciados. Es importante diferenciar los linfomas primarios del tejido mamario de aquellos que se originan en ganglios axilares o intramamarios, así como la infiltración secundaria de la mama por LNH provenientes de otro sitio o por leucemias. La afectación metastásica de este órgano por un tumor primario extramamario se ha reportado en melanomas, carcinomas renales, gástricos y pulmonares, entre otros. Sin embargo, la infiltración linfomatosa de la mama es la neoplasia

(1) Servicio de Oncohematología Hospital General Docente Comandante Pinares, Reparto Noel Camaño, San Cristóbal; Artemisa, Cuba CP24400 *Autor de Correspondencia: gamezoliva1968@gmail.com 
mamaria secundaria más frecuente, aún más que el LNH primario de la mama. En estos casos casi invariablemente los pacientes ya cuentan con una historia previa de LNH diagnosticado y tratado (Ferbeyre-Binelfa et al., 2013; National Cancer Institute, 2015).

En este artículo se presenta un caso de Linfoma no Hodgkin difuso de células pequeñas y grandes hendidas en la mama derecha y se realiza una revisión actualizada del tema.

\section{Caso Clínico}

Paciente de sexo femenino de 72 años de edad que acude a consulta en marzo de 2009 por presentar tumoración de la mama derecha de 3 meses de evolución. Al examen físico presentaba un gran tumor mal delimitado que ocupaba toda la mama, comprometiendo todos los cuadrantes y zona retroareolar, ulcerado, sangrante, con signos inflamatorios. En axila ipsilateral se palpa una adenopatía móvil de $20 \mathrm{~mm}$ de diámetro. Estudio citológico del tumor realizado por punción con aguja fina (PAAF) señala positivo para células neoplásicas.

Se le realiza una biopsia incisional informando linfoma no Hodgkin difuso de células pequeñas y grandes hendidas. Se indica estudio para estadiamiento; Rx de tórax, US de abdomen, TAC de tórax y abdomen y medulograma con biopsia de la médula ósea que todos fueron negativos. Se estadía como una etapa lle. Se indicó tratamiento con quimioterapia 8 ciclos de CHOP (ciclofosfamida, doxorubicina, vincristina y prednisona). Al concluir el segundo ciclo, existía una respuesta de más de un $90 \%$, se continúa tratamiento hasta cumplir el protocolo planificado.

No se indicó radioterapia, porque presentó buena respuesta, con una resolución de un 100\% después del cuarto ciclo. Actualmente está controlada con 5 años de supervivencia libre de enfermedad.

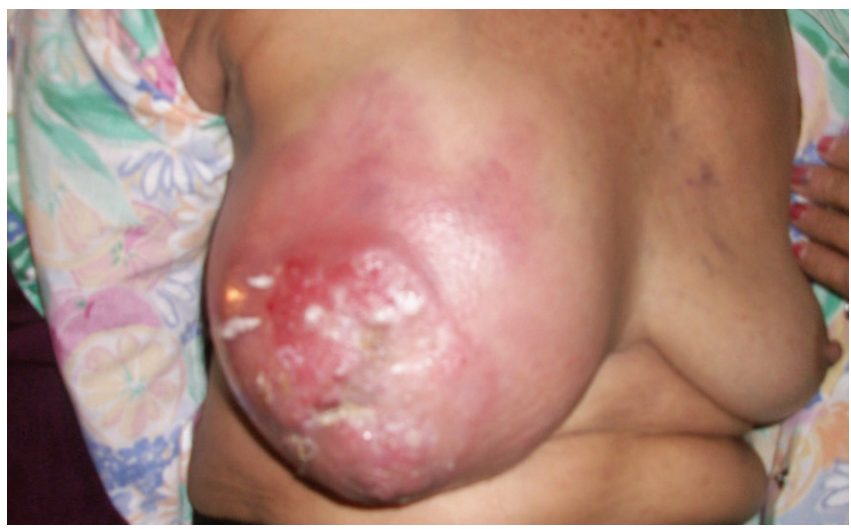

Figura 1. Paciente antes de comenzar el tratamiento. Presentaba un gran tumor mal delimitado que ocupaba todos los cuadrantes y zona retroareolar de la mama derecha, ulcerado, sangrante, con signos inflamatorios. En axila ipsilateral se palpa una adenopatía móvil de $20 \mathrm{~mm}$ de diámetro. Foto aprobada por la paciente para ser publicada con fines científicos.

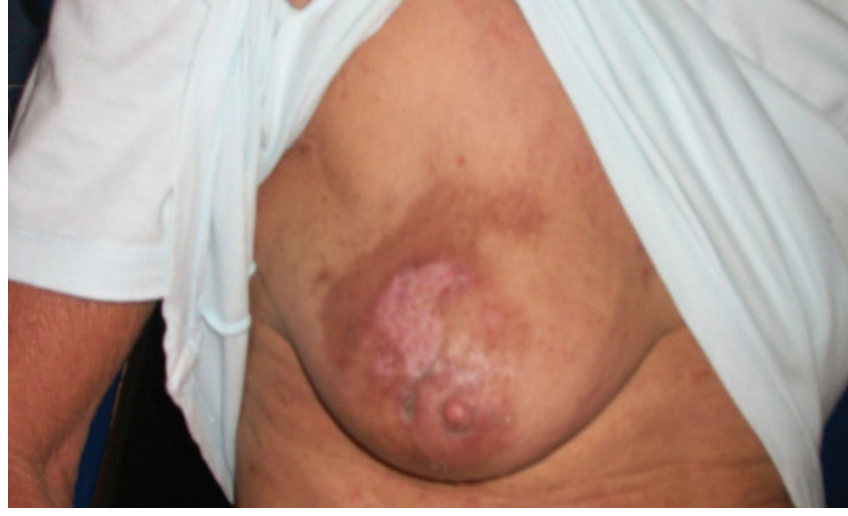

Figura 2. Paciente dos meses después del diagnóstico y haber recibido 2 ciclos de quimioterapia. Se observa regresión de la tumoración en más del 90\% con cicatrización de la lesión ulcerada y desaparición de los signos inflamatorios y la adenopatía axilar derecha. Foto aprobada por la paciente para ser publicada con fines científicos.

\section{Discusión}

El linfoma no Hodgkin primario de la mama (LPM) es una entidad poco frecuente; afecta principalmente a mujeres y representa $0,04 \%$ a $0,5 \%$ de todos los tumores malignos mamarios, aproximadamente el $2 \%$ de los linfomas extranodales y alrededor de 0,5 a $0,7 \%$ de todos los linfomas malignos. La gran mayoría de estos linfomas corresponden al fenotipo celular B (Fajoses Gonçalves et al., 2011; Ferbeyre-Binelfa et al., 2013; Jung et al., 2013).

La edad media de ocurrencia de estos tumores es la quinta década, con un rango de 13 a 88 años. En un estudio realizado donde se revisaron 152 casos la mayoría de las pacientes eran mujeres jóvenes con un promedio de edad de 33 años, hay otras bibliografías donde se presentó con mayor incidencia en pacientes de avanzada edad. Solo se presentó de forma bilateral en un $13 \%$ de los casos donde la población joven fue la mayor afectada (Shim et al., 2013).

En los casos donde existe un compromiso axilar con linfonodos de gran tamaño, con un tumor mamario pequeño localizado cercano a la base de la axila, se debe considerar que el linfoma es de origen nodal y no primario de la mama.

Los criterios diagnósticos originalmente descritos para LPM permanecen siendo la definición de Wiseman, Liao, 1972 (Alves de Souza Scheliga et al., 2012). Estos criterios incluyen:

- La mama tiene que ser el sitio inicial de presentación.

- Ausencia de historia anterior de linfoma.

- Ausencia de enfermedad diseminada al diagnóstico.

- Asociación de linfoma con el tejido mamario en el análisis histopatológico.

- Los linfonodos axilares pueden estar comprometidos desde el mismo momento simultáneo con la mama. Algunos autores 
defienden que cuando los ganglios axilares son voluminosos y tienen mayor volumen que la mama debe ser una extensión secundaria.

El estadiamiento comúnmente utilizado es el sistema Ann Arbor, siendo en las pacientes con LPM comúnmente clasificados como estadio IE (linfonodos axilares negativos) o IIE (linfonodos axilares comprometidos)(Alves de Souza Scheliga et al., 2012).

El linfoma primario de la mama se presenta como un tumor único, indoloro, bien circunscrito, de consistencia gomosa, inmóvil respecto al parénquima adyacente y con rápido crecimiento, con tamaños que van desde los $2 \mathrm{~cm}$ hasta tumores más grandes de 20 $\mathrm{cm}$, con un diámetro promedio de $6 \mathrm{~cm}$. A pesar del gran tamaño que puede alcanzar, no presenta fijación a la pared torácica. Puede existir compromiso de la piel que llegue a la ulceración (linfoma tipo T)(Rathod et al., 2011). El tumor es habitualmente solitario, pero puede asociarse con múltiples nódulos periféricos subcutáneos o a infiltración difusa.

El linfoma primario de mama no presenta características especiales mamográficas o ultrasonográficas que lo distinga de otro tumor de la mama. En la mamografía podemos observar un engrosamiento de la piel y aumento de la opacidad de parénquima, también podemos observar una imagen nodular e irregular que lo hace indistinguible de un proceso neoplásico maligno (Surov et al., 2012). Por otra parte en el estudio ultrasonográfico podemos observar desde imágenes bien definidas a imágenes pobremente definidas (Rathod et al., 2011; Surov et al., 2012).

Es difícil el diagnóstico preoperatorio, la citología mediante BAAF tiene mejor rendimiento que el estudio con material congelado ya que este último tiende a confundirse con el carcinoma (Alves de Souza Scheliga et al., 2012). En un estudio realizado en el INOR, en cuanto al tipo histológico, de una serie de 6 casos predominaron los subtipos histológicos de grado intermedio de malignidad difusos de células grandes y hendidas (Díaz et al., 2001).

En cuanto al tratamiento del linfoma primario de la mama no existe consenso. En series antiguas, décadas de los 70, las pacientes eran sometidas a cirugía y posteriormente a radioterapia; o solamente radioterapia en tumores pequeños obteniendo buen control local de la enfermedad (Alves de Souza Scheliga et al., 2012; Ferbeyre-Binelfa et al., 2013). En una serie de 132 linfomas no Hodgkin primarios de la mama, Fisher y cols., encontraron que el $41 \%$ de las pacientes habían sido sometidas a mastectomía total más disección axilar y el 16\% a mastectomía simple; en un 43\% se realizó una cirugía más conservadora (Fisher \& Chideckel, 1984).
En algunos casos la mastectomía se realizó porque la biopsia contemporánea estableció el diagnóstico de cáncer mamario. Posteriormente se realizaron cirugías más conservadoras como la mastectomía parcial, agregando radioterapia para mejorar el control local de la enfermedad. La mastectomía total se reservó para tumores de gran volumen, infectados o ulcerados (Fisher \& Chideckel, 1984).

Actualmente se orienta el tratamiento sistémico, la quimioterapia, con el objetivo de obtener tanto un control local como también evitar una falla a distancia, con o sin radioterapia, relegando la cirugía para casos especiales; el $80 \%$ de las recurrencias son a distancia. La quimioterapia está indicada especialmente en linfomas con alto grado histológico o intermedio. Hutchings señala obtener buen resultado con la quimioterapia como tratamiento primario o después de una cirugía reductora, con o sin radioterapia regional (Alves de Souza Scheliga et al., 2012; Ferbeyre-Binelfa et al., 2013; Patron \& Miles, 2013; Rivera Mellado et al., 2015).

La sobrevida a 5 años es de $35 \%$ a $64 \%$ y el pronóstico estaría en relación con la edad y estadio de la enfermedad. En la serie de 6 casos presentados por el Dr. Díaz Salas el intervalo libre de enfermedad a 5 años fue del $83 \%$, mientras que la sobrevida global también a 5 años fue del 100 \% (Díaz et al., 2001). En un análisis estadístico de 152 casos de linfoma primario de la mama, en la literatura japonesa, mostró que el tipo histológico, el tipo de tratamiento, y el tamaño tumoral no tuvieron significancia como factor pronóstico (Jeon et al., 1992). En cambio la edad y el estadio fueron predictores estadísticos significativos en la sobrevida. La sobrevida es menor en pacientes jóvenes y la bilateralidad no tendría mayor importancia. Otros, en cambio, citan que el tipo histológico sería un factor pronóstico determinante en la sobrevida, alto grado histológico se asociaría a peor pronóstico (Pandure et al., 2013; Zhong et al., 2014; Chaudhuri et al., 2015; Rivera Mellado et al., 2015).

\section{Conclusiones}

Los LNH primarios de la mama son enfermedades poco frecuentes, pero no excepcionales. Su cuadro clínico y radiológico puede ser muy polimorfo; muchas veces pueden simular una enfermedad benigna y otras veces un carcinoma. El diagnóstico histopatológico se realiza con los mismos criterios que en otros sitios extraganglionares, de acuerdo con la inmunohistoquímica. El tratamiento es muy similar al que se realiza en otros sitios extraganglionares de linfoma. En el orden práctico, la conducta más adecuada es personalizar el tratamiento en cada caso a manera de obtener el máximo beneficio con el mínimo de morbilidad. 


\section{Referencias}

Alves de Souza Scheliga A, Reinert T, Lima Siqueira dos Santos A \& Gustavo Stefanoff C. (2012). Linfoma primario da mama: apresentação clínica e características histopatológicas e moleculares. Revista Brasileira de Oncologia Clínica 8, 79-87.

Chaudhuri A, Samaddar A \& Nag D. (2015). Primary Breast Lymphoma: a rare case report with emphasis on role of cytology, cell block and tru-cut biopsy in diagnosis. 20152.

Díaz CM, Barroso MC, Vásquez E, Expósito G \& Pérez A. (2001). Linfoma de Hodgkin primario de la mama. Presentación de 6 casos y revisión de la literatura. Rev Cub Oncol 17, 210-216.

Fajoses Gonçalves JT, Ribeiro Giordani R, Lemos Lima P, Kapp Rangel K, Lanza de Melo G, de Paula Paim S, de Oliveira Sales PG \& Wagner P. (2011). Linfoma primário de mama: relato de caso. Rev Bras Mastologia 21, 70-72.

Ferbeyre-Binelfa L, Cavazos-Samán C \& Salinas-García JC. (2013). Linfoma no Hodgkin primario de mama: reporte de un caso y revisión de la literatura. Gaceta Mexicana de Oncología 12, 453-458.

Fisher MG \& Chideckel NJ. (1984). Primary lymphoma of the breast. Breast 10, 7-9.

Jeon HJ, Akagi T, Hoshida Y, Hayashi K, Yoshino T, Tanaka T, Ito J, Kamei T \& Kawabata K. (1992). Primary non-Hodgkin malignant lymphoma of the breast. An immunohistochemical study of seven patients and literature review of 152 patients with breast lymphoma in Japan. Cancer 70, 2451-2459.

Jung SP, Kim M, Han KM, Kim J-H, Kim JS, Nam SJ, Bae JW \& Lee JE. (2013). Primary breast lymphoma: a single institution's experience. J Korean Surg Soc 84, 267-272.
National Cancer Institute. (2015). Linfoma no Hodgkin en adultos. Bethesda, MD.

Pandure M, Karle R, Dongre S \& Baviskar B. (2013). Primary non-Hodgkin's lymphoma of the breast: a case report. Internet Journal of Medical Update 8, 34-36.

Patron R \& Miles EF. (2013). Stage IAE Follicular Lymphoma of the Breast: Case Report and Review of the Literature. Case Reports in Oncological Medicine 2013, 3.

Rathod J, Taori K, Disawal A, Gour P, Dhakate S, Mone R \& Bellam KP. (2011). A Rare Case of Male Primary Breast Lymphoma. Journal of Breast Cancer 14, 333-336.

Rivera Mellado VM, Contla Hosking JE, Cuba Velez de Villa R \& C.R. PG. (2015). Linfoma de mama, Informe de un caso. Revista Salud Quintana Roo 8, 19-22.

Shim E, Song SE, Seo BK, Kim Y-S \& Son GS. (2013). Lymphoma Affecting the Breast: A Pictorial Review of Multimodal Imaging Findings. Journal of Breast Cancer 16, 254-265.

Surov A, Holzhausen HJ, Wienke A, Schmidt J, Thomssen C, Arnold D, Ruschke K \& Spielmann RP. (2012). Primary and secondary breast lymphoma: prevalence, clinical signs and radiological features. The British Journal of Radiology 85, e195-e205.

Verdecia-Cañizares C, Português-Díaz A, Alonso-Pirez M \& Cubero Meléndez O. (2011). Linfoma no Hodgkin primario de mama, revisión de la literatura y presentación de un caso. Rev Cub Ped 83.

Zhong J, Di L \& Zheng W. (2014). Synchronous breast cancer and breast lymphoma: two case reports and literature review. Chinese Journal of Cancer Research 26, 355-359. 